\title{
Estado poblacional del cucarachero de pantano, Cistothorus apolinari (Passeriformes: Troglodytidae) en siete humedales de la Sabana de Bogotá, Colombia
}

\author{
Juan Carlos Rodríguez-Linares ${ }^{1}$, Sergio Chaparro-Herrera ${ }^{2 *}$, Adriana Sua-Becerra ${ }^{3}$ \\ \& María Ángela Echeverry-Galvis ${ }^{1}$ \\ 1. Pontificia Universidad Javeriana, Facultad de Estudios Ambientales y Rurales, Bogotá, Colombia; \\ juanrodriguez.linares@gmail.com, ma.echeverryg@javeriana.edu.co \\ 2. Grupo de Ecología y Evolución de Vertebrados, Universidad de Antioquia, Medellín, Colombia; \\ sergioupn@gmail.com \\ 3. Jardín Botánico de Bogotá José Celestino Mutis, Bogotá, Colombia; adrianaupn@gmail.com \\ * Correspondencia
}

Recibido 13-XII-2018. Corregido 27-VIII-2019. Aceptado 18-IX-2019.

\begin{abstract}
Poblational status of the Polinar's Wren, Cistothorus apolinari (Passeriformes: Troglodytidae) in seven wetlands of Bogota Sabanas, Colombia. Introduction: The Apolinar's Wren Cisthotorus apolinari is an endemic species of the Eastern Andes of Colombia currently classified as Critically Endangered (CR) at the national level and Endangered (EN) worldwide, mainly due to the degradation of wetlands, their primary habitat, and the parasitism of the nests. Objetive: Evaluate the state of the populations of C. apolinari in seven wetlands of the Sabana de Bogotá, searching to define what the areas evaluated mean that it hosts the largest population of the species and what other factors determine these population sizes. Methods: between July and December 2014 in seven wetlands we monitoring carried out using counting points and auditory censuses, the abundance of the Apolinar's Wren was recorded, the vegetation cover where the individuals were recorded and the abundances of Shiny Cowbird Molothrus bonariensis. Results: There were $63.6 \mathrm{~h}$ of observation and 88 counting points, obtain a nine records of C. apolinari, one individual in Tibanica, three in La Florida and five in Gualí, mainly associated with the reed Schoenoplectus californicus. None of the biotic and abiotic factors evaluated in the wetlands, were found to determine the presence of the Apolinar's Wren, but trends were present for some variables as the presence of $S$. californicus and Thypa spp. Conclusions: The population of $C$. apolinari has had a significant reduction of up to $94 \%$, added to a possible local extinction in the wetland of La Conejera. These results seek to provide relevant information to contribute to the formulation of effective conservation measures for the protection of the species and its habitat throughout its distribution in the country.
\end{abstract}

Key words: Andean wetlands, Apolinar's Wren, birds, conservation, population monitoring, threatened species.

Rodríguez Linares, J. C., Chaparro-Herrera, S., Sua-Becerra, A., \& Echeverry-Galvis, M. A. (2019). Estado poblacional del cucarachero de pantano, Cistothorus apolinari (Passeriformes: Troglodytidae) en siete humedales de la Sabana de Bogotá, Colombia. Revista de Biología Tropical, 67(6), 1257-1268.

En Colombia y a nivel internacional los ecosistemas dulceacuícolas, cómo los humedales, enfrentan grandes amenazas (SenhadjiNavarro, Ruiz-Ochoa, \& Rodríguez, 2017). Estas áreas cobran relevancia por prestar servicios ecosistémicos, valores biológicos y ser considerados como uno de los ecosistemas más vulnerables frente a los cambios ambientales, además de ser refugio de una gran riqueza de aves (ABO, 2000; Herrera, 
Sepúlveda, \& Aguirre, 2008; Guzmán, 2012; Franco, Delgado, \& Andrade, 2013; ChaparroHerrera, 2015). En Colombia muchas de estas especies se encuentran en alguna categoría de riesgo de extinción como consecuencia de la destrucción de su hábitat principalmente (Hernández, Hurtado, Ortiz, \& Walschburger, 2002; Sua-Becerra \& Chaparro-Herrera, 2015; Renjifo, Amaya-Villarreal, Burbano-Girón, \& Velásquez-Tibatá, 2016). Procesos como la urbanización, ganadería, relleno, vertimiento de aguas residuales, entre otros, modifican las coberturas vegetales originales alterando las condiciones de ciclos y servicios ecosistémicos (ABO, 2000; van der Hammen, 2003; Etter, Mcalpine, Wilson, Phinn, \& Possingham, 2006; Bierwagen, 2007; Guzmán, 2012; Franco et al., 2013).

La Sabana de Bogotá es una altiplanicie ubicada en la cordillera Oriental de Colombia en la cual se encuentran diversos tipos de hábitat como bosques y matorrales nativos y foráneos, potreros, cultivos, humedales, lagos, embalses, estanques, entre otros, que hacen posible el establecimiento o permanencia de más de 400 especies de aves (ABO, 2000; Chaparro-Herrera, Lopera-Salazar, \& Stiles, 2018), que representa cerca del $20 \%$ del total de especies consideradas para Colombia (Avendaño et al., 2017). De estas, alrededor del $6 \%$ se encuentran en las zonas dulceacuícolas, en donde se reporta la extinción del zambullidor andino Podiceps andinus, y 15 en alguna categorías riesgo de extinción para el país, entre las que se encuentra el cucarachero de pantano Cistothorus apolinari (Chapman, 1914) (Passeriformes, Troglodytidae), especie endémica de Colombia y categorizada en Peligro Crítico de extinción (CR) a nivel nacional (ChaparroHerrera et al., 2013; Renjifo et al., 2016) y En Peligro (EN) a nivel mundial (BirdLife International, 2018). Dentro de las principales causas de riesgo están el deterioro y contaminación del hábitat y la creciente presión por parasitismo de nidada por el chamón Molothrus bonariensis (Renjifo et al., 2016; Rosselli et al., 2016; BirdLife International, 2018).
Como parámetro vital para la consideración de categorías de riesgo se encuentra el tamaño poblacional, el cual estaría estrechamente vinculado a la disponibilidad de hábitat con buenas condiciones para el mantenimiento de los ciclos de vida (Renjifo et al., 2016; BirdLife International, 2018). Sin embargo, para este cucarachero, debido a las amenazas documentadas a lo largo de toda su distribución y a posibles cambios taxonómicos como especies separadas en la zona de altiplano y de páramo (en C. a. hernandezi y C. a. apolinari [Stiles \& Caycedo, 2002; Cadena, 2003]), su tamaño poblacional parece continuar en disminución (Sua-Becerra \& Chaparro-Herrera, 2015; Rosselli, Zuluaga-Bonilla, Hernández-Jaramillo, \& Benítez-Castañeda, 2016; Stiles, Rosselli, \& De La Zerda, 2017). Este trabajo evaluó el estado de las poblaciones del cucarachero de Apolinar o de pantano (C. a. apolinari) en siete humedales de la Sabana de Bogotá, buscando definir cuál de las áreas evaluadas alberga la mayor población de la especie y que otros factores determinan dichos tamaños poblacionales, para brindar información en la formulación de medidas de conservación efectivas para su protección y la de su hábitat, ya que es probable que en pocos años la especie desaparezca de los humedales de Bogotá (Renjifo et al., 2016).

\section{MATERIALES Y MÉTODOS}

Área de estudio: La Sabana de Bogotá hace parte de la región sur del altiplano Cundiboyacense sobre la cordillera Oriental de Colombia. Presenta una temperatura media anual de $13{ }^{\circ} \mathrm{C}$, precipitación de $866 \mathrm{~mm}$ y altura promedio de $2650 \mathrm{~m}$ (Guhl, 1981; ABO, 2000). Originalmente se encontraba bosque alto andino, páramo, matorrales xerofíticos y humedales (CAR, 2000), donde actualmente domina la matriz urbana de Bogotá D. C., con humedales remanentes aislados, entre una extensión de $40 \mathrm{~km}$ en sentido Norte-Sur y de $20 \mathrm{~km}$ en sentido Oriente-Occidente que es la ciudad (Calvachi, 2003). 
Muestreo: Se seleccionaron siete humedales completamente rodeados por condiciones urbanas, que tenían registros históricos de $C$. apolinari (ABO, 2000; Morales-Rozo, 2001; Rosselli \& Stiles, 2012, Chaparro-Herrera, 2015), así como áreas que ofrecían accesibilidad a los muestreos y acompañamiento institucional público o privado. Los humedales seleccionados fueron: Guaymaral, La Conejera, Juan Amarillo, Jaboque, La Florida, Gualí y Tibanica (Tabla 1, Fig. 1). Estos fueron visitados entre los meses de julio y diciembre de 2014 , donde se realizaron 35 visitas y 88 puntos de conteo sin radio fijo. Estos puntos estaban distanciados entre si un mínimo de 150 metros, para abarcar el mayor perímetro, área y hábitats posibles en cada humedal (Ralph et al., 1996). La abundancia fue estimada a partir de la sumatoria de individuos por punto de censo en cada humedal. En cada humedal se realizaron visitas y puntos de censo de la siguiente manera, de acuerdo al área total de cada uno: Guaymaral: cinco visitas, siete puntos (julio a octubre y diciembre). La Conejera: cinco visitas, 15 puntos (julio y octubre). Juan Amarillo: cuatro visitas, 12 puntos (julio y noviembre). Jaboque: seis visitas, 14 puntos (julio y noviembre). La Florida: cinco visitas, 12 puntos (agosto y noviembre). Gualí: cinco visitas, 17 puntos (agosto y diciembre), y Tibanica: cinco visitas, 11 puntos (julio y diciembre). En cada visita se ubicaron los mismos puntos de muestro por medio de georreferenciación. El muestreo fue realizado entre las 06:00 h y las 12:00 h con observaciones directas, mismo observador, con la ayuda de binoculares 8 x $42 \mathrm{~mm}$ por un periodo de diez minutos por punto, durante los cuales se registraba la presencia y abundancia de la especie, así como aspectos comportamentales (canto, forrajeo, percha, interacciones y descripción de otro tipo de actividad observada). Pasado este tiempo en cada punto de censo y si la especie no se había detectado, se realizaba un "playback", reproduciendo los cantos y llamados de la especie con grabaciones obtenidas de la página www.xeno-canto.org (número XC117660, XC117649) y del archivo de la guía sonora de los Andes colombianos (Álvarez-Rebolledo, Caro, Laverde, \& Cuervo, 2007), por un periodo de un minuto, esperando respuesta visual o auditiva. Se prestó particular atención a la presencia o no del parasito de

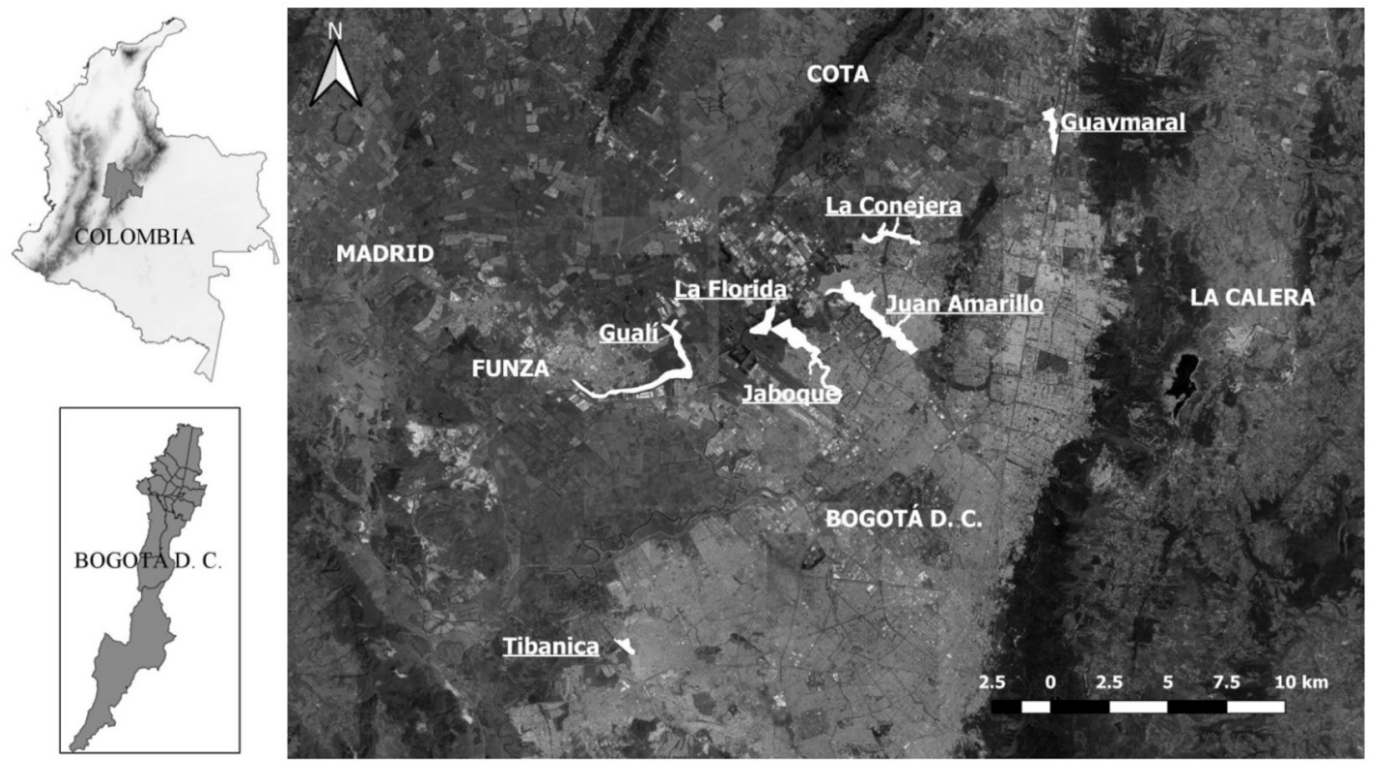

Fig. 1. Humedales presentes en la Sabana de Bogotá seleccionados para determinar el estado poblacional de Cistothorus apolinari.

Fig. 1. Wetlands in the Sabana de Bogotá selected to determine the population status of Cistothorus apolinari. 
TABLA 1

Humedales en la Sabana de Bogotá seleccionados para determinar el estado poblacional de Cistothorus apolinari

TABLE 1

Wetlands in the Sabana de Bogotá selected to determine the population status of Cistothorus apolinari

\begin{tabular}{|c|c|c|c|}
\hline Humedal & Coordenadas & Extensión (ha) & Ubicación \\
\hline Guaymaral & $4^{\circ} 48^{\prime} 17.7^{\prime \prime} \mathrm{N}-74^{\circ} 2^{\prime} 30.6^{\prime \prime} \mathrm{W}$ & 37.9 & Localidad de Suba, Bogotá \\
\hline La Conejera & $4^{\circ} 45^{\prime} 40.3^{\prime \prime} \mathrm{N}-74^{\circ} 6{ }^{\prime} 21.6^{\prime \prime} \mathrm{W}$ & 64 & Localidad de Suba, Bogotá \\
\hline Juan Amarillo & $4^{\circ} 43^{\prime} 6.3^{\prime \prime} \mathrm{N}-74^{\circ} 5 ' 56.7^{\prime \prime} \mathrm{W}$ & 231 & Localidad de Suba y Engativá, Bogotá \\
\hline Jaboque & $4^{\circ} 43^{\prime} 20.1^{\prime \prime} \mathrm{N}-74^{\circ} 8^{\prime} 41.1^{\prime \prime} \mathrm{W}$ & 308 & Localidad de Engativá, Bogotá \\
\hline La Florida & $4^{\circ} 43^{\prime} 35.3^{\prime \prime} \mathrm{N}-74^{\circ} 9^{\prime} 0.1{ }^{\prime \prime} \mathrm{W}$ & 31 & Municipios de Funza y Cota, Cundinamarca \\
\hline Gualí & $4^{\circ} 42^{\prime} 23.5^{\prime \prime} \mathrm{N}-74^{\circ} 10^{\prime} 46.4^{\prime \prime} \mathrm{W}$ & 100.7 & Municipio de Funza y Mosquera, Cundinamarca \\
\hline Tibanica & $4^{\circ} 36^{\prime} 13.3^{\prime \prime} \mathrm{N}-74^{\circ} 12^{\prime} 19.8^{\prime \prime} \mathrm{W}$ & 16.6 & Localidad de Bosa, Bogotá \\
\hline
\end{tabular}

cría chamón Molothurs bonarienses, en caso de su detección se hizo una cuantificación de individuos dado que está contemplado como una de las principales amenazas para $C$. apolinari (Velásquez-Tibatá, Gutiérrez, \& Carrillo, 2000; Caycedo \& Renjifo, 2002; MoralesRozo \& De La Zerda, 2004; Villaneda-Rey \& Rosselli, 2011).

Coberturas vegetales: Se definieron cinco unidades de cobertura vegetal (sensu IGAC, 1994), con la adición de una basada en características históricas de uso de la especie en los humedales, así como con zonificaciones recientes (ABO, 2000; Morales-Rozo, 2001; Secretaria Distrital de Ambiente, 2008; Rosselli \& Stiles, 2012) (Tabla 2), estas coberturas fueron corroboradas en visitas previas de campo. A partir de imágenes satelitales aportadas por el software Google Earth Pro. 7.1.5, se exportaron en máxima definición a ArcGIS 10.3 a escala 1:1 000, con una escala grafica de salida entre 1:2 500 a 1:14 000. Los contornos creados, se delimitaron, georreferenciaron, y digitalizaron por cada una de las categorías de cobertura definidas. Se cuantificó el área total de trabajo, el área por cobertura y el perímetro de cada humedal.

TABLA 2

Descripción de las unidades de cobertura vegetal en siete humedales de la Sabana de Bogotá

TABLE 2

Description plants cover units in seven wetlands in the Sabana de Bogotá

\begin{tabular}{|c|c|c|}
\hline Cobertura & Nombre & Descripción vegetación \\
\hline CV1 & junco & Dominada por Schoenoplectus californicus. \\
\hline CV2 & enea & Dominada por Typha latifolia y Typha angustifolia. \\
\hline CV3 & junco-enea & $\begin{array}{l}\text { Sin dominancia aparente y presentando una asociación vegetal de Schoenoplectus } \\
\text { californicus y Typha latifolia. }\end{array}$ \\
\hline CV4 & vegetación flotante & $\begin{array}{l}\text { Dominada por: Baccharis latifolia (chilco), Polygonun hydropiperoides (hierba de } \\
\text { sapo), Rumex conglomeratus (lengua de vaca), Limnobium laevigatum (buchón), } \\
\text { Scenecio carbonelli (margarita de pantano), Bidens laevis (botoncillo) y Lemna sp. } \\
\text { (lenteja de agua). }\end{array}$ \\
\hline CV5 & vegetación de ronda & $\begin{array}{l}\text { Dominada por: Salix humboldtiana (sauce), Alnus acuminata (aliso), } \\
\text { Sambucus peruviana (sauco), Polymnia pyramidalis (arboloco), Fraxinus uhdei (F. } \\
\text { chinensis) (urapán), Eucalyptus globulus (eucalipto) y Pennisetum clandestinum } \\
\text { (kikuyo). }\end{array}$ \\
\hline C6 & espejo de agua & Medida que se delimita a $15 \mathrm{~m}$ desde donde termina el cuerpo de agua. \\
\hline
\end{tabular}


Análisis estadístico: Se cuantificó cuántas y cuáles coberturas vegetales usó C. apolinari, para conocer si la especie mantenía la preferencia o uso de hábitat que se halló en estudios anteriores en la Sabana de Bogotá (MoralesRozo, 2001; Rosselli \& Stiles, 2012). Del total de puntos de censo, se realizó una sumatoria de los sitios de presencia por cada cobertura, en relación al porcentaje de disponibilidad total de cada cobertura para los humedales estudiados. Esto se complementó con un análisis de preferencia de hábitat o recursos siguiendo a Montenegro y Acosta (2008) mediante la cuantificación del número de veces que se observó en cada cobertura, sobre el número total de puntos realizados. Debido al elevado número de puntos sin detección de la especie, el censo proporcionó una gran cantidad de datos de cero (0), lo cual impidió realizar una regresión lineal por violación de supuestos de homocedasticidad y distribución normal de los residuos (Gotelli \& Ellison, 2004), por lo cual los datos se evaluaron como presencia-ausencia de registros de $C$. apolinari. Se realizaron regresiones logísticas para determinar qué variables influenciaron la probabilidad de encontrar a C. apolinari en cada área, utilizando como variables predictoras el porcentaje de extensión de cada cobertura vegetal, porcentaje de representación del espejo de agua, área, perímetro del humedal y el promedio de individuos de Molothrus bonariensis. Los análisis fueron realizados usando la función glm en R v3.1.3 (R Core Team, 2015).

\section{RESULTADOS}

Con un total de $63.6 \mathrm{~h}$ de observación se obtuvieron nueve registros de $C$. apolinari (siete visuales y dos auditivos) entre agosto y noviembre: un individuo en Tibanica, tres individuos en La Florida y cinco individuos en Gualí. Esto es equivalente al $6.17 \%$ de detección en los puntos del estudio. Dado que los individuos no fueron capturados y marcados de manera individual para determinar si los avistamientos realizados en meses diferentes corresponden o no a individuos diferentes, se presume que esta especie es bastante fiel a sus sitios y territorios (Morales-Rozo \& De La Zerda, 2004; Renjifo et al., 2016), por lo cual cada individuo visto en cada censo se tomó como un individuo, a menos que claramente se observaran más de uno (o se observara y escuchara otro). Mediante la fotointerpretación, se identificó que no todos los humedales contenían todas las coberturas. En los humedales de Guaymaral, Gualí y Tibanica el área fue dominada por CV1, siendo en Gualí y Tibanica más del $50 \%$ del área total de cada humedal, esta cobertura se presentó en todos los humedales evaluados. En Juan Amarillo, no se encontró la cobertura CV2 y en Jaboque, La Florida y Gualí ésta fue menor al $1 \%$ del área del humedal, solo en Guaymaral esta cobertura presentó un mayor valor con $18.2 \%$. La cobertura vegetal $\mathrm{CV} 3$ presentó valores menores al $7 \%$ en cinco de los siete humedales, siendo menor al $1 \%$ en La Conejera. En La Florida y Gualí presentó valores superiores con 21 y $13.7 \%$ respectivamente. Por su parte CV4, se presentó en todos los humedales con dominancia en Jaboque y La Conejera, donde se encontró en más del $40 \%$ de la extensión, siendo menor en La Florida con 8 \%. El espejo de agua abierto (C6), si bien también presente en todos los humedales fue muy reducido en Jaboque (3\%), con mayor cobertura el Juan Amarillo (55.2\%) y La Florida (31.4 \%) (Tabla 3).

TABLA 3

Porcentaje de cinco coberturas vegetales y de espejo de agua a partir de fotointerpretación en siete humedales de la Sabana de Bogotá (ver Tabla 2 para definiciones)

TABLE 3

Percentage of five plants covering and open water from photointerpretation in seven wetlands in the Sabana de Bogotá (see Table 2 for descriptions)

\begin{tabular}{lcccccc}
\multicolumn{1}{c}{ Humedal } & CV1 & CV2 & CV3 & CV4 & CV5 & C6 \\
Tibanica & 59.85 & 8.3 & 3.02 & 25.4 & 3.2 & 2.1 \\
Gualí & 62.3 & 0.9 & 13.7 & 12.1 & 4 & 2.4 \\
Florida & 22.3 & 0.9 & 21.4 & 8.5 & 17.2 & 31.4 \\
Jaboque & 16.2 & 0.3 & 3.1 & 75.0 & 3.0 & 3.1 \\
Juan Amarillo & 12.6 & 0 & 2.03 & 11.4 & 17.3 & 55.2 \\
La Conejera & 12.5 & 6.1 & 0.8 & 42.2 & 26.4 & 11.2 \\
Guaymaral & 41.7 & 18.5 & 6.2 & 15 & 6.1 & 12.2 \\
\hline
\end{tabular}


TABLA 4

Análisis de preferencia de hábitat. Porcentajes de oferta de hábitat y preferencia en las coberturas vegetales presentes y usadas por Cistothorus apolinari en siete humedales de la sabana de Bogotá

TABLE 4

Habitat preference analysis. Percentage of habitat offer and preference in plan coverings present and used by Cistothorus apolinari in seven wetlands in the Sabana de Bogotá

\begin{tabular}{|c|c|c|c|c|c|c|}
\hline & CV1 & CV2 & $\mathrm{CV} 3$ & CV4 & CV5 & C6 \\
\hline Puntos de censo con presencia de cada cobertura / Puntos totales & $47 / 88$ & $13 / 88$ & $24 / 88$ & $53 / 88$ & $31 / 88$ & $23 / 88$ \\
\hline Observaciones de C. apolinari/Obs. totales & 06-sep & 01 -sep & 02-sep & 0 & 0 & 0 \\
\hline Porcentaje de disponibilidad en el área de estudio (\%) & 53.40 & 14.70 & 27.20 & 60.20 & 35.20 & 26.10 \\
\hline Porcentaje de Uso (\%) & 11.23 & 6.80 & 7.33 & 0 & 0 & 0 \\
\hline
\end{tabular}

C. apolinari fue observado utilizando las coberturas como sitios de percha, vocalización $\mathrm{y}$ alimentación y sin registros de actividad reproductiva (elaboración de nidos o cría de polluelos). En el humedal de La Florida se observó en el junco (CV1) a dos individuos solitarios, uno en enea (CV2) y otro en la cobertura mixta de junco-enea (CV3), todos los registros hacia la zona sureste del humedal. En Gualí se observaron tres individuos (grupo) en junco (CV1) hacia el extremo norte del humedal, y una pareja en la mezcla de junco y enea (CV3) hacia la parte más sur, y al igual que en el anterior humedal, los registros fueron entre los meses de agosto y noviembre. Finalmente en Tibanica el único individuo observado se encontró entre juncos (CV1), hacia la entrada del humedal en la parte suroeste.

Como una aproximación a la preferencia de hábitat, se encontró que si bien la cobertura con mayor presencia en los 88 puntos de censo fue la vegetación flotante (CV4) con un 60.2 $\%$ de disponibilidad del total de los humedales, esta no fue utilizada por la especie (Tabla 4). La cobertura vegetal junco (CV1) fue la segunda más abundante, en 47 de los 88 puntos de censo (Tabla 4), siendo la más usada por $C$. apolinari con seis puntos de censo con detección, lo cual equivale a $11.2 \%$ de uso. La siguiente cobertura vegetal en preferencia de uso fue la mezcla de junco-enea (CV3) con un $7.33 \%$, y por último enea (CV2) con $6.79 \%$ (Tabla 4).

Respecto a la presencia de M. bonariensis, se encontró entre 0.9 y 2.61 individuos por hectárea; el humedal Gualí presentó el mayor número de individuos totales con 593 a lo largo del muestreo, pero también el mayor número en una sola visita con un valor aproximado de 260 individuos. En La Conejera se registró el segundo mayor número de individuos en un muestro con 150 individuos; para los siete humedales visitados en promedio el número de chamones no estuvo nunca por debajo de los 45 individuos. A partir de la regresión logística de presencia o ausencia de $C$. apolinari en relación con las variables seleccionadas, no fue posible identificar alguna que determinará la presencia de la especie (Tabla 5). Bajo esta regresión, las variables podrían tener mayor influencia serían

\section{TABLA 5}

Regresión logística de las variables estudiadas en relación con la presencia de Cistothorus apolinari en siete humedales de la Sabana de Bogotá

TABLE 5

Logistic regression of the variables studied in relation to the presence of Cistothorus apolinari in seven wetlands in the Sabana de Bogotá

\begin{tabular}{cccc} 
Variable & Estimates (b) & Error estándar & $\operatorname{Pr}(>|\mathrm{z}|)$ \\
CV1 & 0.213 & 0.021 & 0.062 \\
CV2 & -0.023 & 0.823 & 0.076 \\
CV3 & 0.071 & 0.028 & 0.323 \\
CV4 & -0.295 & 0.003 & 0.534 \\
CV5 & -0.421 & 0.619 & 0.214 \\
C6 & -0.097 & 0.194 & 0.634 \\
Área & -0.004 & 0.281 & 0.823 \\
Perímetro & 0.028 & 0.005 & 0.421 \\
M. bonariensis & 0.084 & 0.176 & 0.087 \\
\hline
\end{tabular}

Estimates (b): intercepto estimado; $\operatorname{Pr}(>|z|)$ : Probabilidad estimada. 
la cobertura de junco (CV1) y de enea (CV2), donde la presencia de $M$. bonariensis podría ser otra variable para monitorear.

\section{DISCUSIÓN}

A lo largo de todo el muestreo se encontraron nueve individuos de C. apolinari, con registros en tres de los siete humedales evaluados. Estos registros se realizaron principalmente en coberturas de junco (Schoenoplectus californicus) y de enea (Typha spp.), y aunque se detectó que la especie muestra preferencia por dichas coberturas entre las disponibles en cada humedal, no se encontró una variable vegetal o física del humedal que pudiera contribuir significativamente con su presencia en los mismos.

En una perspectiva histórica sobre el estado poblacional de $C$. apolinari, se tiene un primer registro metódico de Morales-Rozo (2001) quien encontró 27 individuos (18 en Tibanica, 6 en La Conejera y 3 en La Florida). En 2012 Rosselli y Stiles (2012) reportaron para La Conejera 2 individuos, Juan Amarillo 5 , Tibanica 5 y Gualí 20. Estos últimos reportes históricos constituirían los más altos a la fecha, indicando en comparación con este estudio, que las poblaciones de este cucarachero son susceptibles de fluctuaciones en los humedales estudiados. La población de Tibanica presentaría, en este lapso de tiempo, una posible reducción del $94 \%$, la del humedal de Gualí $75 \%$, mientras que en relación a los individuos del humedal Juan Amarillo, no es posible hacer la misma comparación pues por cuestiones de logística en este trabajo solo se censó el tercio alto, mientras en el trabajo de Rosselli y Stiles (2012) en todo el humedal se reportaron cinco individuos, por lo cual no se puede aseverar que esta especie no se encuentre hoy en día en esta localidad. Para humedal La Conejera, los muestreos se realizaron en recorridos similares a los de Morales-Rozo (2001) y Rosselli y Stiles (2012), por lo que podría considerarse una extinción local de la especie en dicho humedal. Por su lado la población que parece ser más estable, pero con un número muy bajo de individuos, es La Florida.

Al comparar los 27 individuos reportados por Morales-Rozo (2001), los 32 individuos de Rosselli y Stiles (2012), con los nueve individuos del presente estudio, se pone de manifiesto que la población de los humedales de la Sabana de Bogotá tiene una tendencia a la reducción desde el 2001 (Morales-Rozo, 2001; Rosselli \& Stiles, 2012), con una posible disminución del $85.1 \%$ de la población. Si bien las metodologías en cada estudio difirieron en sus recorridos específicos, teniendo en cuenta que en todas se reportó número de individuos totales, esto puede ser una forma de aproximarse a estas comparaciones. Al ser C. apolinari una especie altamente territorial y sedentaria (Kroodsma et al., 1999; Morales-Rozo, 2005; Morales-Rozo \& De La Zerda, 2004; Renjifo et al., 2016), podría indicar que la especie este sufriendo extinciones puntuales, en lugar de considerar posibles oportunidades de dispersión por entre una matriz altamente inhóspita.

Morales-Rozo (2001) reportó de forma exclusiva a $C$. apolinari sobre junco en todos los humedales, lo cual difiere del presente trabajo para La Florida, en donde se observó el uso de tres coberturas diferentes: junco, enea y junco-enea; mientras que en Tibanica la cobertura de uso fue nuevamente junco. En cuanto a lo reportado por Rosselli y Stiles (2012) solo se presentó cambio en el humedal Gualí, en este se encontró el uso de la cobertura junco-enea. Las coberturas vegetales en el humedal de Guaymaral, La Conejera, Jaboque y Tibanica, y sus dominancias, fueron similares a lo encontrado por Rosselli y Stiles (2012), pero en Juan Amarillo y Gualí la dominancia de estas cambió, pudiendo relacionarse con la limitación de muestreos al tercio alto del humedal debido a condiciones de seguridad. La cobertura más dominante fue el espejo de agua, seguido de vegetación de ronda y el junco, difiriendo a lo encontrado por Rosselli y Stiles (2012), los cuales reportan la vegetación flotante como la más abundante. En Gualí, la cobertura dominante, con más del $50 \%$ del área, fue junco, seguido de la asociación vegetal junco-enea, 
mientras que en 2012, la vegetación flotante era la más dominante seguida del junco (Rosselli \& Stiles, 2012). Esto podría responder en alguna medida al manejo activo que se ha realizado en los diferentes humedales por diferentes entidades a cargo de estas zonas (como la Empresa de Acueducto y Alcantarillado de Bogotá y la Corporación Autónoma Regional de Cundinamarca), en cuanto la remoción de cobertura vegetal flotante. En Gualí, se observó una extensa intervención mecánica en la extracción de vegetación flotante durante el periodo de estudio con retroexcavadora, lo cual pudo haber modificado las proporciones de las coberturas, llevando a un aumento de junco y enea, favoreciendo a la población de C. apolinari en este humedal. A primera vista, esto podría parecer una intervención positiva para la especie de cucarachero, sin embargo hasta donde esto pueda ser extendido en otros humedales queda por definir, pues previo al muestreo se presentó en Tibanica la remoción de lodos del canal adyacente a este, actividad similar a la desarrollada en Gualí, pero que en este caso llevó posiblemente, a una pérdida de cobertura de junco (J. Rodríguez Linares obs. pers.).

Solo para tres humedales es posible hacer una comparación directa en relación con las coberturas de 2001 (Morales-Rozo, 2001) con el número de individuos de la especie de interés, a pesar que en dicho trabajo no se realizó cuantificación de las coberturas, sin embargo mediante la interpretación de la cartografía presentada en encontramos que en La Conejera se encuentra que se mantiene la vegetación flotante como la cobertura dominante, mientras que para la Florida y Tibanica se encuentran cambios con una dominancia del espejo de agua en lugar de junco en donde en el último la cobertura más dominante actual es espejo de agua versus junco, mientras que en el humedal La Florida el espejo de agua sigue siendo la más dominante, con aumento en junco como la segunda más abundante y no la vegetación flotante. Estos cambios en dominancia de las coberturas podrían estar relacionado con cambios temporales dentro del régimen del sistema como por ejemplo por el asentamiento de la vegetación, la cual tiene fundamento en las fluctuaciones climáticas (Andrade, 1998), el manejo particular que se realiza a cada uno de los humedales, la calidad de agua (Castro et al., 2005), y la cercanía de vías y centros poblados (Etter et al., 2006). Si C. apolinari requiere de coberturas específicas que se están viendo alteradas por las modificaciones paisajísticas, sus poblaciones serán cada vez menores.

Los bajos números de $C$. apolinari en la última década y el continuo descenso de sus poblaciones se postulan están relacionados directamente con la degradación y perdida de su hábitat (Rosselli et al., 2016), de manera conjunta con otras amenazas, dentro de las cuales se encuentra el parasitismo social por parte de $M$. bonariensis, fenómeno que reduce el éxito reproductivo de las ya diezmadas poblaciones de C. apolinari (Morales-Rozo, 2005; Villaneda-Rey \& Rosselli, 2011; Rosselli et al., 2016). Durante este trabajo no se observaron eventos de parasitismo directo o de alimentación de juveniles de $M$. bonariensis por parte de C. apolinari, pero si sobre Zonotrichia capensis, en los mismos puntos donde se observó C. apolinari, particularmente en La Florida. A diferencia de Villaneda-Rey y Rosselli (2011), en los humedales La Conejera, Guaymaral, Tibanica y Gualí, el número de individuos por hectárea de $M$. bonariensis fue reportado entre 0.32 y 0.50 , mientras que en este estudio para los mismos humedales, se encontraron entre 0.9 y 2.61 individuos por hectárea, con un mayor incremento en La Conejera donde el número pudo haberse quintuplicado. Villaneda-Rey y Rosselli (2011), ya hacían referencia a que dado el bajo número de $C$. apolinari, la presencia de unos pocos M. bonariensis podría ser devastadora para la supervivencia de esta especie, pero con la cantidad de $M$. bonariensis encontrados en este estudio, sería de esperarse que su contribución en procesos de declive poblacional de C. apolinari sea aún más contundente.

En el modelo de regresión con las cinco coberturas vegetales evaluadas, porcentaje de espejo de agua, área, perímetro del humedal y promedio de individuos de $M$. bonariensis, no 
se encontró ninguna variable significativamente relacionada o no con la presencia de C. apolinari, en donde por ejemplo se encontró tanto en humedales de área pequeña (16.65 ha) como Tibanica, hasta humedales de área grande como Gualí (100.71 ha). En humedales con diferentes porcentajes de abundancia para la cobertura vegetal clave para la especie como el junco (Schoenoplectus californianus), como en el humedal Gualí con el 62.6 \% y La Florida con el $23.2 \%$; esta variable tampoco fue indicativa de presencia, aun cuando esta podría tener algún soporte estadístico como influyente en la determinación de la presencia de la especie. En los valores de la regresión logística, tampoco se encontró relación entre $C$. apolinari y la presencia de $M$. bonariensis, sin que esto permita descartar de inmediato que la interacción con esta especie no sea un factor determinante de la baja abundancia y presencia. Si se considera que la regresión se realizó bajo las condiciones actuales, es muy difícil definir de qué forma las interacciones pasadas entre las especies fueron las que contribuyeron específicamente en los resultados de números de individuos a la fecha. Más aun, cuando la abundancia de $C$. apolinari ya era baja en lo reportado por Morales-Rozo (2001), donde la única relación encontrada fue el tamaño del parche de junco, de entre 0.05 a 5 ha y alturas de este mayores a dos metros, tamaños que encontramos en los sitios de estudio y en los cuales no se encontró la especie. Incluso Rosselli y Stiles (2012), con números más elevados de individuos, y con 28 variables ambientales y 19 humedales, definen que la presencia de $C$. apolinari, estaría relacionada con alrededor de 13 variables conjuntas, entre ellas la relación área/perímetro, las coberturas vegetales y abundancia de $M$. bonariensis, mostrando que $C$. apolinari es una especie que requiere de alta especificidad de hábitat.

C. apolinari se encuentra afectado por procesos acorde al paradigma de las poblaciones pequeñas, en los cuales, procesos de autoecología, endogamia, cuellos de botella y deriva génica (Caughley, 1994), generarían eliminación completa de poblaciones, como lo observado en las poblaciones del cóndor californiano
(Gymnogyps californianus), donde su continuo descenso poblacional hasta de 19-21 individuos en 1983, generó la necesidad de manejo en cautiverio con herramientas genéticas para su recuperación en vida silvestre (Ralls \& Ballou, 2004). Queda la pregunta abierta sobre cuál sería la capacidad de movilidad de esta especie entre posibles hábitats fuentes y sumideros, y si es que hubiera opción de abordar su conservación en el marco de metapoblaciones (Theodorou, Souan, \& Couvet, 2009; Bergerot, Julliard, \& Baguette, 2010; Mouquet, Matthiessen, Miller, \& González, 2011), basados en el principio de conectividad funcional, que ha sido poco estudiada en estos ecosistemas urbanos (Haig, Mehlman, \& Oring, 1998). Sería importante considerar que dada la cercanía geográfica entre los humedales de Gualí-La Florida-Jaboque $(3.0 \mathrm{~km}$ lineales entre Gualí y La Florida, $1.1 \mathrm{~km}$ entre la zona de registros en La Florida y Jaboque), e incluso Juan Amarillo (a $2.2 \mathrm{~km}$ lineales de Jaboque), estos tres humedales podrían actuar bajo un marco no solo metapoblacional sino de metacomunidades (Leibold et al., 2004), que permitiría la conservación de la especie, pero esto queda por definirse por metodologías de seguimiento telemétrico o estructuras genéticas.

Dados los resultados obtenidos en este estudio, hacemos un llamado urgente a conocer el estado poblacional de esta especie en otras áreas de su distribución para poder determinar las acciones específicas en cada área; dado que de no tomarse medidas de inmediato la especie podría extinguirse en pocos años. Importante conocer los estados poblacionales de C. a. apolinari en la laguna de Fúquene (Cundinamarca) y Tota (Boyacá), en donde se han calculado cerca de 200 y 356 individuos respectivamente (Rosselli et al., 2016), siendo la mayor concentración de individuos remanentes, números que igualmente están muy por debajo de dar alguna tranquilidad en procesos poblacionales. Esta información debería lleva a acciones de conservación inmediatas dirigidas a la especie. Por un lado, la protección estricta de sitios con tamaños poblacionales muy bajos, en donde se pueda hacer una restauración activa encaminada 
a aumentar las zonas de juncos y enea, y por otro, en aquellas localidades con mayor número de individuos, propender activamente por el incremento de las coberturas de preferencia de la especie, a la vez que se desarrolla trabajo de apropiación y valoración por parte de las comunidades humanas aledañas. Esto dado que se hacen algunos usos no sostenibles de estas especies vegetales que pueden afectar sus procesos de establecimiento y reproducción. Igualmente, mantener el monitoreo sobre las interacciones de especies invasoras como el chamón, con el cucarachero y otras aves, para definir si fuera necesario medidas de control población a este parasito de cría. Estas acciones no solo beneficiarían a C. a. apolinari, sino que actuando como especie sombrilla beneficiaria a otras que habitan humedales en el altiplano Cundiboyacense, y que también presentan alguna categoría de amenaza nacional como la tingua bogotana Rallus semiplumbeus, el pato turrio Oxuyra jamaicensis, el doradito oliváceo Pseudocolopteryx acutipennis, la tingua de pico verde o moteada Porphyriops melanops, entre otras.

Declaración de ética: los autores declaran que todos están de acuerdo con esta publicación y que han hecho aportes que justifican su autoría; que no hay conflicto de interés de ningún tipo; y que han cumplido con todos los requisitos y procedimientos éticos y legales pertinentes. Todas las fuentes de financiamiento se detallan plena y claramente en la sección de agradecimientos. El respectivo documento legal firmado se encuentra en los archivos de la revista.

\section{AGRADECIMIENTOS}

A Neotropical Bird Club por la financiación de este trabajo mediante sus premios de conservación. A la Empresa de Acueducto y Alcantarillado de Bogotá (EAAB) y el Instituto Distrital de Recreación y Deporte (IDRD) por el permiso de ingreso. A L. M. Renjifo y L. Rosselli por comentarios en versiones iniciales de este estudio y manuscrito. A tres revisores anónimos por sus aportes y comentarios, así como a D. Santos y D. Rodríguez por observaciones complementarias en campo. Finalmente a M. Echeverry por su ayuda en la fotointerpretación.

\section{RESUMEN}

Introducción. Cisthotorus apolinari es una especie endémica de Colombia actualmente catalogada en Peligro Crítico de extinción nacionalmente, y En Peligro globalmente, principalmente por la degradación de los humedales y el parasitismo de las nidadas. Hay pocos los estudios sobre el estado poblacional de esta especie en los humedales de Bogotá en los últimos años. Objetivo. Evaluar el estado de las poblaciones de C. apolinari en siete humedales de la Sabana de Bogotá, buscando definir cuál de las áreas evaluadas alberga la mayor población de la especie y qué otros factores determinan dichos tamaños poblacionales. Métodos: Entre julio y diciembre 2014 realizamos un monitoreo en siete humedales. Mediante puntos de conteo y censos auditivos registramos la abundancia, las coberturas vegetales empleadas y la abundancia de Molothrus bonariensis. Resultados: Se realizaron 63.6 horas de observación en 88 puntos de conteo, obtuvimos nueve registros de C. apolinari, un individuo en Tibanica, tres en La Florida y cinco en Gualí, asociados principalmente a junco Schoenoplectus californicus. No se encontró ninguna variable en las coberturas vegetales significativamente relacionada o no con la presencia de $C$. apolinari pero se plantean tendencias en algunas variables como la presencia de junco y enea Typha spp. Conclusiones: La población de C. apolinari ha tenido una reducción significativa de hasta el $94 \%$, sumado a una posible extinción local en el humedal de La Conejera. Estos resultados buscan proporcionar información relevante para aportar en la formulación de medidas de conservación efectivas para la protección de la especie y de su hábitat.

Palabras clave: aves, conservación, cucarachero de Apolinar, especies amenazadas, humedales alto andinos, monitoreo poblacional.

\section{REFERENCIAS}

ABO. (2000). Aves de la Sabana de Bogotá, Guía de Campo. Bogotá: Asociación Bogotana de Ornitología (ABO)-CAR.

Álvarez-Rebolledo, M., Caro, V., Laverde, O., \& Cuervo, A. (2007). Guía sonora de las aves de los Andes colombianos. Villa de Leyva, Colombia: Instituto de Investigación en Recursos Biológicos Alexander von Humboldt. 
Andrade, G. I. (1998). Los humedales del altiplano de Cundinamarca y Boyacá. Ecosistemas en peligro de desaparecer. En E. Guerrero (Ed.), Una aproximación a los humedales de Colombia (pp. 59-72). Bogotá: Fondo FEN-Colombia, Comité Colombiano de la UICN, UICN-Oficina Regional para América del Sur.

Avendaño, J. E., Bohórquez, E. I., Rosselli, L., ArzuzaBuelvas, D., Estela, F. A., Cuervo, A. M., Stiles, F. G., \& Renjifo, L. M. (2017). Lista de chequeo de las aves de Colombia: una síntesis del estado del conocimiento desde Hilty \& Brown (1986). Ornitología Colombiana, 16, eA01.

Bergerot, B., Julliard, R., \& Baguette, M. (2010). Metacommunity Dynamics: Decline of Functional Relationship along a Habitat Fragmentation Gradient. PLOS ONE, 5(6), e11294.

Bierwagen, B. G. (2007). Connectivity in urbanizing landscapes: The importance of habitat configuration, urban area size, and dispersal. Urban Ecosystems, 10(1), 29-42.

BirdLife International. (2018). Cistothorus apolinari. The IUCN Red List of Threatened Species 2018. e.T22711366A131963740 (Base de datos). Recuperado de http://dx.doi.org/10.2305/IUCN.UK.2018-2. RLTS.T22711366A131963740

Cadena, C. D. (2003). Taxonomía de Cistothorus apolinari (Troglodytidae), conceptos de especie y conservación de las aves amenazadas de Colombia: un comentario. Ornitología Colombiana, 1, 71-75.

Calvachi, B. (2003). La fauna de los humedales de Bogotá y la Sabana. En A. Guarnizo, \& B. Calvachi (Eds.), Los humedales de Bogotá y la Sabana (pp. 109-140). Bogotá: Empresa de Acueducto y Alcantarillado de Bogotá, Conservación Internacional Colombia.

CAR. (2000). La Estructura Ecológica Principal de la Sabana de Bogotá. Bogotá: Sociedad Geográfica de Colombia, Academia de Ciencias Geográficas, Corporación Autónoma Regional de Cundinamarca (CAR).

Castro, F. L., Cruz, I., \& Moreno, L. A. (2005). Evaluación de la calidad del agua y diagnóstico ambiental del humedal Jaboque. Tecnogestion, 2(1), 77-83.

Caughley, G. (1994). Directions in conservation biology. The Journal of Animal Ecology, 63(2), 215-244.

Caycedo, P., \& Renjifo, L. M. (2002). Cistothorus apolinari. En L. M. Renjifo, A. M. Franco-Maya, J. D. Amaya-Espinel, G. H. Kattan, \& B. López-Lanús (Eds.), Libro rojo de aves de Colombia. Serie Libros Rojos de Especies Amenazadas de Colombia (pp. 379-382). Bogotá: Instituto de Investigación de Recursos Biológicos Alexander von Humboldt y Ministerio del Medio Ambiente.
Chaparro-Herrera, S. (2015). Avifauna presente en 14 Parques Ecológicos Distritales de Humedal (PEDH) de Bogotá. En S. Chaparro-Herrera, \& D. Ochoa (Eds.), Aves de los Humedales de Bogotá, Aportes para su Conservación (pp. 80-91). Bogotá: Asociación Bogotana de Ornitología (ABO).

Chaparro-Herrera, S., Echeverry-Galvis, M. Á., CórdobaCórdoba, S., \& Sua-Becerra, A. (2013). Listado actualizado de las aves endémicas y casi-endémicas de Colombia. Biota Colombiana, 14(2), 235-272.

Chaparro-Herrera, S., Lopera-Salazar, A., \& Stiles, F. G. (2018). Aves del departamento de Cundinamarca: conocimiento, nuevos registros y vacíos de información. Biota Colombiana, 19(1), 160-189.

Etter, A., Mcalpine, C., Wilson, K., Phinn, S., \& Possingham, H. (2006). Regional patterns of agricultural land use and deforestation in Colombia. Agriculture, Ecosystems and Environment, 114, 114-369.

Franco, L., Delgado, J., \& Andrade, G. (2013). Factores de la vulnerabilidad de los humedales altoandinos de Colombia al cambio climático global. Revista Colombiana de Geografia, 22(2), 69-85.

Gotelli, N. J., \& Ellison, A. M. (2004). A primer of ecological statistics. Massachusetts, USA: Sinauer Associate.

Guhl, E. (1981). La sabana de Bogotá, sus alrededores y su vegetación. Bogotá: Jardín Botánico José Celestino Mutis-Instituto Geográfico Agustín Codazzi.

Guzmán, A. (2012). Plantas de los humedales de Bogotá y del Valle de Ubaté. Bogotá: Fundación HumedalesInstituto de Recursos Biológicos Alexandre von Humboldt-Fondo Hugo de Vires.

Haig, S. M., Mehlman, D. W., \& Oring, L. W. (1998). Avian Movements and Wetland Connectivity in Landscape Conservation. Conservation Biology, 12(4), 749-758.

Hernández, J., Hurtado, A., Ortiz, R., \& Walschburger, T. (2002). Vulnerabilidad y estrategias para la conservación de algunos biomas de Colombia. En G. Halffter (Ed.), La Diversidad Biológica De Iberoamérica I (pp. 191-202). México: Instituto de Ecología Xalapa.

Herrera, M. A., Sepúlveda, M. V., \& Aguirre, N. (2008). Análisis sobre la aplicabilidad de las herramientas de gestión ambiental para el manejo de los humedales naturales interiores de Colombia. Gestión y Ambiente, 11(2), 7-25.

IGAC. (1994). Memorias del Primer Taller sobre Cobertura Vegetal, Clasificación y Cartografia - Proyecto SIGPAFC y el INADE. Bogotá: Instituto Geográfico Agustín Codazzi.

Kroodsma, D., Da Silva, M., Goodwin, E., Sánchez, J., Stemple, D., \& Vielliard, J. (1999). Sedentary life 
style of neotropical Sedge Wrens promotes song imitation. Animal Behaviour, 57(4), 855-863.

Leibold, M. A., Holyoak, M., Mouquet, N., Amarasekare, P., Chase, J. M., Hoopes, M. F., Holt, R. D., Shurin, J. B., Law, R., Tilman, D., Loreau, M., \& González, A. (2004). The metacommunity concept: a framework for multi-scale community ecology. Ecology Letters, $7,601-613$

Montenegro, J., \& Acosta, A. (2008). Programa innovador para evaluar uso y preferencia de hábitat. Universitas Scientiarum, 13(2), 208-217.

Morales-Rozo, A. (2001). Caracterización y uso de hábitat del cucarachero de pantano Cistothorus apolinari en los humedales de La Conejera, la Florida, Tibanica, laguna de Fúquene y laguna de Chisacá, Cundinamarca (Tesis de pregrado). Pontificia Universidad Javeriana, Colombia.

Morales-Rozo, A. (2005). Notas Sobre Los Sitios De Anidación Del Cucarachero De Pantano (Cistothorus apolinari). Boletín SAO, 15(2), 61-68.

Morales-Rozo, A., \& De La Zerda, S. (2004). Caracterización y uso de hábitat del Cucarachero de Pantano Cistothorus apolinari (Troglodytidae) en humedales de la Cordillera Oriental de Colombia. Ornitología Colombiana, 2, 4-18.

Mouquet, N., Matthiessen, B., Miller, T., \& González, A. (2011). Extinction Debt in Source-Sink Metacommunities. PLoS ONE, 6(3), e17567.

R Core Team. (2015). $R$ : A language and environment for statistical computing (Software). Vienna: R Foundation for Statistical Computing. Recuperado de http:// www.Rproject.org

Ralls, K., \& Ballou, J. D. (2004). Genetic Status and management of California Condors. The Condor, 106(2), 215-228.

Ralph, C. J., Geupel, G. R., Pyle, P., Martin, T. E., DeSante, D. F., \& Mila, B. (1996). Manual de métodos de campo para el monitoreo de aves terrestres. Albany, USA: Pacific Southwest Section, Forest Service, U.S. Department of Agriculture.

Renjifo, L. M., Amaya-Villarreal, A. M., Burbano-Girón, J., \& Velásquez-Tibatá, J. (2016). Libro rojo de aves de Colombia, Volumen II: Ecosistemas abiertos, secos, insulares, acuáticos continentales, marinos, tierras altas del Darién y Sierra Nevada de Santa Marta, y bosques húmedos del centro, norte y oriente del país. Bogotá, Colombia: Editorial Pontificia Universidad Javeriana e Instituto Alexander von Humboldt.

Rosselli, L., \& Stiles, F. G. (2012). Local and landscape environmental factors are important for the conservation of endangered birds in a high Andean plateau. Waterbirds, 35(3), 453-469.
Rosselli, L., Zuluaga-Bonilla, J. E., Hernández-Jaramillo, A., \& Benítez-Castañeda, H. D. (2016). En L. M. Renjifo, A. M. Amaya-Villarreal, J. Burbano-Girón, \& J. Velásquez-Tibatá (Eds.), Libro rojo de aves de Colombia, Volumen II: Ecosistemas abiertos, secos, insulares, acuáticos continentales, marinos, tierras altas del Darién y Sierra Nevada de Santa Marta y bosques húmedos del centro, norte y oriente del país (pp. 345-351). Bogotá, Colombia: Editorial Pontificia Universidad Javeriana e Instituto Alexander von Humboldt.

Secretaria Distrital de Ambiente. (2008). Protocolo de recuperación y rehabilitación ecológica de humedales en centro urbanos. Bogotá: Dirección de Planeación y Gestión Ambiental, oficina de Ecosistemas Estratégicos y Biodiversidad, Alcaldía Mayor de Bogotá D. C.

Senhadji-Navarro, K., Ruiz-Ochoa, M. A., \& Rodríguez, J. P. (2017). Estado ecológico de algunos humedales colombianos en los últimos 15 años: Una evaluación prospectiva. Colombia Forestal, 20(2), 191-200.

Stiles, F. G., \& Caycedo, P. (2002). A new subspecies of Apolinar's Wren Cistothorus apolinari, (Aves:Troglodytidae), an endangered Colombian endemic. Caldasia, 24(1), 191-199.

Stiles, F. G., Rosselli, L. \& De La Zerda, S. (2017). Changes over 26 Years in the Avifauna of the Bogotá Region, Colombia: Has Climate Change Become Important? Frontiers in Ecology and Evolution, 5, $1-21$.

Sua-Becerra, A., \& Chaparro-Herrera, S. (2015). Aves endémicas, amenazadas, extintas y escapadas. En S. Chaparro-Herrera, \& D. Ochoa (Eds.), Aves de los Humedales de Bogotá, Aportes para su Conservación (pp. 26-38). Bogotá: Asociación Bogotana de Ornitología (ABO).

Theodorou, K., Souan, H., \& Couvet, D. (2009). Metapopulation persistence in fragmented landscapes: significant interactions between genetic and demographic processes. Journal of Evolutionary Biology, $22,152-162$.

van der Hammen, T. (2003). Los humedales de la Sabana: origen, evolución, degradación y restauración. En A. Guarnizo, \& B. Calvachi (Eds.), Los humedales de Bogotá y la Sabana (pp. 19-51). Bogotá: Acueducto de Bogotá, Conservación Internacional Colombia.

Velásquez-Tibatá, J., Gutiérrez, A., \& Carrillo, E. (2000). Primer registro de parasitismo reproductivo en el Cucarachero de Pantano Cistothorus apolinari por el Chamón Maicero Molothrus bonariensis. Cotinga, 14, 102 .

Villaneda-Rey, M., \& Rosselli, L. (2011). Abundancia del Chamón Parásito (Molothrus bonariensis, Icteridae) en 19 humedales de la Sabana de Bogotá. Ornitología Colombiana, 11, 37-48. 